\title{
Self-study colaborativo: etapas, condiciones de la amistad crítica y aprendizajes sobre la formación
}

\author{
Alejandra Nocetti de la Barra' (D) (a) \\ Claudia Orrego Lepe ${ }^{2}$ (D) @ \\ Ilich Silva-Peña ${ }^{3}$ (1) @ \\ David Santibáñez Gómez ${ }^{4}$ (D) @ \\ Marianella Flores González ${ }^{5}$ (D) (a) \\ Marlene Gutiérrez Villegas ${ }^{6}$ (D) @ \\ Pablo Moreno Tello ${ }^{7}$ (D) @ \\ Romina González Sáez ${ }^{1}$ (1) @
}

${ }^{1}$ Universidad Católica Santísima Concepción; ${ }^{2}$ Universidad Católica de Temuco; ${ }^{3}$ Universidad de Los Lagos; ${ }^{4}$ Universidad Finis Terrae; ${ }^{5}$ Universidad de Talca; ${ }^{6}$ Universidad Academia de Humanismo Cristiano; ${ }^{7}$ Universidad Católica Silva Henríquez .

Resumen. En el presente artículo abordamos el trabajo realizado por un grupo de académicos utilizando la metodología de investigación denominada self-study. El fin investigativo fue abordar las etapas de este método reflexivo y crítico sobre el 'sí mismo', las condiciones de la amistad crítica y el aprendizaje en la formación de formadores. Examinamos la enseñanza de la reflexión en actividades curriculares de índole práctica en dos universidades, constituyendo trabajos en parejas. Un/a integrante desarrollaba el self-study, mientras otro/a ejercía el rol de amigo/a crítico/a. Analizamos correos electrónicos, notas de diarios, conversaciones grabadas, filmaciones de clases y discusiones. Como resultado de este proceso, determinamos que desde el punto de vista metodológico hubo distinciones en las etapas del self-study, así como consideraciones de importancia en la construcción de la amistad crítica para el desarrollo de este tipo de investigación. Por último, discutimos los hallazgos en torno a dos tipos de aprendizaje consumados, a saber, sobre los formadores de formadores y los estudiantes que, de modo indirecto, acompañaron el proceso de cambio de los docentes en estudio.

Palabras clave: formador de docentes; análisis cualitativo; Chile; cooperación intelectual.

Self-study colaborativo: etapas, condições da amizade crítica e aprendizagem sobre a formação Resumo. No presente artigo, abordamos o trabalho realizado por um grupo de acadêmicos utilizando a metodologia denominada autoestudo. O objetivo da pesquisa foi abordar as etapas deste método reflexivo e crítico sobre o 'si mesmo', as condições da amizade crítica e a aprendizagem na formação de formadores. Examinamos o ensino da reflexão em atividades curriculares de índole prática em duas universidades, constituindo trabalhos em pares. Um/a integrante realizava o self-study, enquanto outrola exercia o papel de amigo/a crítico/a. Analisamos e-mails, anotações de diários, conversas gravadas, vídeos feitos em sala de aula e discussões. Como resultado deste processo, determinamos que, do ponto de vista metodológico, houve diferenças nas etapas da realização do self-study, assim como considerações importantes na construção da amizade crítica para a realização deste tipo de pesquisa. Por último, discutimos as descobertas sobre os dois tipos de aprendizagem consumados sobre os formadores de formadores e os estudantes que indiretamente acompanharam o processo de mudança dos docentes analisados.

Palavras-chave: formador de docentes; análise qualitativo; Chile; cooperação intelectual.

Collaborative Self-Study: stages, conditions of critical friendship and learning about training Abstract. In this article, we describe a collaborative Self-Study carried out by teacher educators who intentionally examined their teaching of reflection. The research purpose was to approach the stages of this method of reflective and critical style on the 'self,' the conditions of critical friendship and learning in the formation of teacher educators. We examined the teaching of reflection in practical courses in two Chilean university institutions, constituting collaborative pair-work; where one of the members developed the Self-Study and the other was the one who played the role of a critical friend. Besides, interuniversity meetings were held and the results, difficulties, and preliminary conclusions discussed. We analyzed the exchanges of emails, journal notes, recorded conversations, filming in class, and discussions of the work couples and the interuniversity group. As a result, we found that there were distinctions upon the methodological point of view when facing the different stages of the Self-Study development, besides the importance of the construction of critical friendship 
for the development of this type of research emerged as essential — finally, findings regarding the learning experience as teacher educators as well as the learning from the students perspective arose. Keywords: teacher educators; qualitative analysis; Chile; intellectual cooperation.

\section{Introducción}

En este artículo, presentamos parte de los resultados de una investigación colaborativa interuniversitaria apoyada por la Organización de Estados Iberoamericanos (OEI). El objetivo que nos propusimos en este proyecto fue impulsar el desarrollo de estudios colaborativos focalizados en la formación del profesorado a través del uso de la metodología del self-study a nivel nacional. En particular, constituimos este equipo interinstitucional a partir del llamado realizado por la OEI. Aunque el proceso de construcción de relaciones en el grupo de investigación lo desarrollamos en un artículo anterior (Silva-Peña et al. 2017), contextualizarermos el proceso de convergencia que llevó a este estudio.

El grupo de investigadores/as conformado, carecía de nexos comunes. Si bien, algunos de los partícipes compartíamos docencia o gestión, en una misma universidad, la relación a través de la investigación era nula entre nosotros. Por tanto, creamos un grupo heterogéneo, donde participamos investigadores con experiencia en proyectos y publicaciones, así como colegas que consideraban que su labor estaba volcada principalmente a la docencia y en el caso de la investigación su rol era el de "aprendiz". Siendo un punto relevante para esta investigación la conformación de un equipo proveniente de diversas instituciones, ubicadas en tres regiones de Chile.

El proyecto de investigación exigía una problematización, un punto común de partida, por tanto, nuestros procesos de reflexión en la formación docente se manifestaron como una gran problemática a desarrollar. Así, con la reflexión transformada en nuestro telón de fondo, dimos origen al intercambio de conocimientos y saberes pedagógicos (Contreras, 2011; 2013 y Contreras y Pérez de Lara, 2010) construidos por los propios participantes, Ilevando el foco del trabajo en torno a los dispositivos utilizados en clases para provocar la reflexión en docentes en formación. Cabe señalar que algunos de los investigadores habíamos estudiado la reflexión docente (Silva-Peña, Valenzuela y Santibáñez, 2008; Nocetti, 2016; Nocetti y Medina, 2017; 2018; 2019) o teníamos conocimiento sobre la problemática de la práctica reflexiva en la formación inicial (Chacón y González, 2008; Correa, Chaubet, Collin y Gervais, 2014; Guerra, 2009; Korthagen, 2010; Russell, 2012; Hirmas y Cortés, 2015; Del Barrio, 2014). Aun así, el trabajo con la metodología selfstudy , fue algo nuevo (Silva-Peña et al. 2017; Loughran y Russell, 2016; Berry, 2016; Russell, Fuentealba, e Hirmas, 2016). 
El intercambio de experiencias en las sesiones de trabajo en la OEI, nos llevó de forma paulatina a un giro epistemológico (Perrenoud, 2004; Tardif, 2004; Schön, 1992). Dejamos de poner el foco en el estudio de la experiencia del otro/a, volcando la mirada hacia nuestra propia práctica formativa. Desde ahí, levantamos nuestra propia problematización. Dicho problema, de forma inicial, quedó circunscrito a estudiar los dispositivos docentes para provocar la reflexión. A poco andar entenderíamos la esencia del self-study cuyo centro está en los cambios de las propias prácticas docentes, en el ejercicio de los formadores/as de formadores/as. Es así, que este artículo comparte los resultados de las experiencias vividas con la metodología self-study al examinar/indagar de modo intencional la propia práctica formativa referida al aprendizaje y enseñanza de la reflexión en el contexto de la práctica pedagógica en dos instituciones universitarias del sur de Chile.

\section{Marco teórico}

Nuestro trabajo como formadores de docentes en aulas universitarias y el conocimiento que se desprende de investigaciones recientes, ha reforzado en nuestro equipo el valor que tiene promover la experiencia reflexiva durante la formación profesional. La práctica reflexiva, cuenta con variadas ventajas, entre estas: subraya el carácter deliberativo de la enseñanza, estimula la construcción de saberes pedagógicos, articula la teoría aprendida en la universidad y lo vivido en la experiencia. Además, desarrolla la habilidad para cambiar y explorar nuevas formas de actuación profesional (Altet, 2010). De este modo, el ejercicio de la reflexión temprana, en docentes en formación, favorecería la construcción de conocimiento práctico, devolviendo el valor a lo vivido, en la perspectiva del desarrollo profesional en la docencia (Paquay et al. 2010; Tardif, 2004; Perrenoud, 2004; 2010).

También, el conocimiento práctico, entendido como aquel que se origina en el ejercicio de la docencia, dirigiendo qué hacer en situaciones formativas (Contreras, 2016; Alliaud y Suárez, 2011; Tardif, 2004), nos muestra que, a pesar de reconocer la importancia de la práctica reflexiva, aún quedan interrogantes respecto a cómo desarrollar esta habilidad en el profesorado; relevando el debate sobre el predominio de un modelo de formación que promueve la memorización de contenidos sin lograr el desarrollo del pensamiento crítico (OCDE, 2004, 2009).

En este caso, entendemos que la experiencia docente al ser analizada de modo reflexivo, moviliza la construcción de saberes profesionales que articulan tanto, lo que trae el sujeto (desde su propia biografía escolar), lo que aporta el proceso formativo y lo que le ocurre en términos profesionales 
en la institución universitaria y en la escuela (Contreras 2011, 2013; Tardif, 2004). Todos estos elementos, si son tomados como objeto de reflexión, tienen un alto potencial de aprendizaje para el futuro docente; y en esta perspectiva, pensamos que la metodología self-study es al mismo tiempo un enfoque de investigación y una oportunidad para construir conocimientos prácticos y transformadores de manera colaborativa (Loughran y Russell, 2016).

Entendemos al self-study como una metodología que nos permite estudiar la práctica desarrollada por los/as mismos docentes (Hamilton, 1998). "self-study es una posibilidad real de construir conocimiento a partir de nuestro propio quehacer pedagógico. Una metodología que es familiar puesto que observamos desde nuestro rol formador, permitiendo investigar el accionar docente desde la cotidianeidad de enseñar." (Silva-Peña, et al., $2017,120-121)$. Se trata de una investigación personal de la propia práctica (Berry, 2016). Para nosotros como formadores de formadores/as, también significa una metodología que desplaza algunos paradigmas de la tradición científica, permitiendo unir investigación y formación desde la propia comprensión de nuestro self (sí mismo). Un tipo de investigación que permite la creación de comunidades al interior de los equipos de formación de docentes (Samaras, 2010).

Como formadores/as de formadores/as, tenemos un alto compromiso con el desarrollo de una "práctica reflexiva" temprana en la formación docente, lo que resulta complejo por varias razones. Una, tiene que ver con la falta de acuerdo respecto del significado de la reflexión docente (Nocetti, 2016; Nocetti y Medina, 2019) y por consiguiente, didácticamente suele ser difícil proponer modelos de trabajo que desarrollen esta habilidad, complejizando la certeza de que estamos estudiando la reflexión y no un constructo similar.

Atendiendo a lo anterior, es pertinente aclarar el concepto de "práctica reflexiva" utilizada en este self-study. En primer lugar, recalcar que una práctica reflexiva no se logra con un cuestionamiento esporádico de la acción profesional, sino que requiere de un proceso que forma parte del habitus del docente (Perrenoud, 2010), asumiendo que existe un conocimiento en la acción que emerge de la experiencia reflexionada, vinculándola así a la "reflexión sobre y en la acción" (Schön, 1992).

La reflexión sobre la acción es una actividad mental que toma la propia acción como objeto de reflexión, ya sea para valorarla o someterla a crítica en términos técnicos, prácticos o emancipatorios. Esta reflexión, permite anticipar una acción futura o simularla a través del pensamiento, constituyendo un proceso mental producido durante una acción profesional, particularmente, al enfrentar un problema que no logra resolverse con el conocimiento que se tiene en un momento determinado (Perrenoud, 2004). 
Estas situaciones tienen una función crítica, poniendo en duda el conocimiento profesional y disponiendo a la reestructuración de estrategias y la comprensión misma de la profesión (Schön, 1992).

Cabe subrayar que como formadores/as de formadores/as aspiramos a promover una experiencia de reflexión con carácter práctico/crítico que inste al profesorado a reconocer los supuestos y consecuencia éticas de la misma (Grundy, 1987; Silva-Peña y Paz-Maldonado, 2019). Por otra parte, y de acuerdo a las condiciones institucionales actuales, es difícil promover una reflexión de carácter crítica pues la política pública ha mermado la capacidad del profesorado para analizar críticamente la realidad escolar, dejando en el campo del olvido la responsabilidad social y política de los profesionales de la educación.

De este modo, antecedentes contextuales, teóricos y empíricos, sumados a las percepciones acerca del trabajo docente, generan preocupación acerca del acto reflexivo al interior del aula universitaria. Aunque hay diferencias en torno a las experiencias académicas, desarrollos teóricos y uso de ciertos dispositivos, se configura como problemático el saber cómo mejorar la enseñanza de la reflexión. De ahí que esta investigación la centremos en la propia acción docente y transite por un paradigma específico (Guba y Lincoln, 1994), a fin de responder a la naturaleza de la cuestión (Connelly y Clandinin, 1990), en cuanto a: ¿Qué nos ocurre al examinar/indagar de modo intencional la propia práctica formativa referida al aprendizaje y enseñanza de la reflexión mediante la metodología self-study en el contexto de las asignaturas de prácticas pedagógicas?

\section{Metodología}

El desarrollo de la investigación tuvo un enfoque interpretativo orientado a comprender, desde la perspectiva de los sujetos- formadores de formadores-, la experiencia referida al aprendizaje de enseñar a enseñar en una perspectiva reflexiva (Álvarez-Gayou, 2013; Crotty, 1988; Sandin, 2003). De modo particular, el estudio refiere a lo cualitativo, pues es la propia experiencia formativa -el sí mismo-, donde el docente se transforma en objeto/sujeto de estudio que potencia procesos de aprendizaje tanto en su formación como en la de los estudiantes a cargo. Esta investigación, privilegió la construcción de significado producto de la interacción social y la interpretación en conjunto con otros para dar sentido a la acción humana en el contexto de la formación inicial docente (Bisquerra, 2014; Rodríguez, Gil y García, 1999; Sandin, 2003). 
Self-study, es parte de las denominadas metodologías de investigación docente (Silva-Peña, 2007). En este caso, debe comprenderse como una indagación intencional y sistemática de la propia práctica formativa, con el fin de mejorarla y a la vez transformarnos. Como equipo, consideramos la propuesta de self-study que acuña Berry (2016), quien lo comprende como un proceso investigativo de carácter personal acerca de la propia práctica como formador o formadora de docentes. Al mismo tiempo, valoramos el aporte de Loughran y Russell (2016), quienes definen esta experiencia, de carácter colaborativo, como contrapunto a la investigación tradicional sobre la enseñanza, ya que valora la perspectiva de los propios formadores y avanza hacia el reconocimiento de la enseñanza como una disciplina independiente.

Nos inspiran en autores como Clandinin y Connelly (2007) o Tardif (2004), quienes recalcan la importancia de estimular el levantamiento del conocimiento que está presente en la experiencia de los y las formadoras de formadores. Dicho conocimiento debiera constituirse como una experiencia formativa compartida entre docentes universitarios con el fin de esclarecer la forma de mejorar la efectividad de nuestro quehacer formativo (Loughran, 2007). De este modo, el self-study como experiencia- de investigación y autoformación- desarrolla una aproximación sobre el self (sí mismo), en el contexto de enseñanza y aprendizaje de la reflexión en el taller de práctica pedagógica.

\subsection{Constitución del equipo self-study}

En Chile la realización de self-studies tiene una historia muy reciente, tanto en el ámbito investigativo como en la asignación de un estilo de trabajo colectivo e interinstitucional. El equipo participante estuvo compuesto por ocho académicos provenientes de tres universidades. Pensamos la organización del grupo en parejas de trabajo, donde uno de los integrantes analizaba su práctica y otro tomaba el rol de amigo crítico en la experiencia. Cada par, documentaba el trabajo de investigación de su práctica de modos diversos (grabaciones, textos y videos) y compartíamos los hallazgos parciales en reuniones ampliadas. Por la distancia en la que nos encontrábamos utilizábamos video conferencia, documentos compartidos, correo electrónico, entre otros medios tecnológicos.

Este tipo de self-study, planteado como colaborativo interinstitucional, para nosotros constituyó un doble desafío. Por una parte, nos permitió mirar nuestro propio quehacer como objeto y sujeto a la vez. Por otra pate, facilitó el compartir experiencias intersubjetivas, a través de las miradas externas de la amistad crítica. 


\subsection{Recogida de los datos}

Como equipo, nos costó liberarnos de la epistemología de las evidencias y del "dato duro". Caminamos generando una amplia gama de herramientas que permitieran sustentar la mirada hacia las propias prácticas reflexivas, obviando, algunas veces, la propia perspectiva del desarrollo de self-study. Desde esa mirada, tratamos de resguardar la visión de los tres actores -formador de formadores, estudiantes y amigo crítico, utilizando distintas técnicas de recolección según los actores involucrados (tabla 1). Todas, según conveniencia de cada pareja, las describimos brevemente a continuación:

Tabla 1. Descripción de las estrategias de producción de evidencias

\begin{tabular}{lll}
\hline \multicolumn{1}{c}{ Formador de formadores } & Amigo crítico & Estudiantado \\
\hline Diario docente & - Escritura de correos electrónicos & Diario docente \\
& - Conversaciones de investigación & \\
& - Observación de videos de clases &
\end{tabular}

Fuente: Elaboración propia en función de la experiencia de los participantes.

\subsection{Diario docente}

El diario docente, insumo reconocido como estrategia de producción de dato cualitativo en el contexto de la investigación social, vincula recursos que favorecen los procesos de reflexión sobre nuestra propia práctica (Pozo, 2012; Sanjurjo, 2005; Zabalza, 2005). El uso de este, tuvo dos sentidos; por un lado, actuar como recurso didáctico para promover la reflexión en el taller de práctica con estudiantes de pedagogía y por otro, constituir una estrategia de recogida de información por parte del formador sobre su propia experiencia reflexiva.

\subsection{Correos electrónicos}

El correo electrónico tuvo como fin recoger la relación intersubjetiva mediada por la escritura: levantando datos, saberes y aprendizajes respecto de nuestra experiencia formativa compartida, examinada, reflexionada e interpretada de cara a un desarrollo profesional. Lo consideramos una experiencia narrativa sobre lo vivido (Contreras, 2013; Connelly y Clandinin, 1990). De manera particular, nuestro self-study trabajó el correo electrónico con una frecuencia que fue variando de lo semanal a lo quincenal, conforme los acontecimientos y la experiencia avanzaba. 


\subsection{Conversaciones de investigación}

Esta técnica de investigación, permitió desarrollar conversaciones semanales con el amigo crítico, constituyéndose dicha instancia comunicativa en una estrategia de construcción de textos de campo (Clandinin y Connelly 2000; Silva-Peña, Driedger-Enns, Huber y Houle, 2016). En las conversaciones sostenidas con él o la amiga crítica, notamos dos niveles de obtención de datos. Al inicio, compartimos lo que nos ocurría, haciendo centro en lo aparente y con un enfoque descriptivo. Pero, con el tiempo, advertimos que volcamos las conversaciones hacia los significados presentes y transformativos, sobre lo vivido en el aula, haciendo foco en dato textual-personal del cambio, con alto potencial de interpretación sobre lo sucedido.

\subsection{Observación de videos de clases}

En la trama de esta experiencia de self-study empleamos la noción de observación participante, ya que no se trata de observar para "chequear/ verificar/confirmar", sino de centrarse en un fragmento de lo ocurrido en la clase universitaria y a partir de esto, examinar lo sucedido para extraer los significados que subyacen a nuestras prácticas formativas. Esta información, de tipo cualitativa se transforma en el sustrato desde el cual emergen los hallazgos que se presentarán posteriormente. Por consiguiente, la observación de segmentos de videos de clases permitió profundizar en la comprensión de lo vivido en las aulas. Y, desde esta perspectiva, esta recogida de datos al entrelazarse con la función del amigo crítico configuró espacios para aumentar la conciencia sobre lo que hacemos y sus consecuencias, resultando clave en la construcción de conocimiento sobre el proceso formativo de este self-study.

\subsection{Análisis y hallazgos}

Los análisis de los documentos emanados los hicimos a través de una lectura crítica de diversas fuentes seleccionadas. Los textos en un comienzo los volcamos a un documento de forma dispersa y, poco a poco, fuimos dando paso a un documento en el que construimos categorías a partir de lo observado. Dichas categorías, basadas en las experiencias individuales, las fuimos unificando en el transcurso de la discusión. Para la elaboración de este artículo, incorporamos las categorías resultantes de nuestros aprendizajes como formadores/as. 


\section{Hallazgos}

Los distintos self-studies realizados nos arrojaron una diversidad de hallazgos. Si embargo, por un sentido de focalización y de espacio, hemos organizado los resultados en 4 temáticas que consideramos relevantes para este artículo, puesto que son los que generaron mayor resonancia de modo colectivo:

1. La experiencia con la metodología self-study con carácter colaborativo e interinstitucional

2. El proceso de construcción de la amistad crítica como elemento parte del mismo estudio

3. Los aprendizajes construidos como formadores/as de formadores/as y

4. Aprendizajes desde los/as mismos/as estudiantes recogidos a través de los diversos self-study

\subsection{Una mirada a la experiencia de investigar mediante self-study}

A través del análisis de los textos emanados, escribimos un primer documento en el que señalaban diversos aprendizajes en medio del trabajo de investigación (Silva-Peña, et al. 2017). Luego de un análisis más detallado nos dimos cuenta que existían ciertas etapas en este proceso de aprendizaje.

Al observar nuestro trabajo, en retrospectiva, logramos reconocer cuatro etapas durante el desarrollo del self-study y subrayamos que este hallazgo, fue una cuestión compartida y reconocida por cada uno de los formadores de formadores que participamos de este proyecto interuniversitario de investigación.

\section{Etapa 1. Resistencia al foco en lo subjetivo.}

Con esto, nos referimos al momento vivido por quienes participamos del proyecto; pues al inicio y de modo general, existía una resistencia (o tal vez incapacidad) a mirar nuestro "propio yo", como objeto/sujeto de estudio. La mirada estaba puesta primordialmente en la efectividad de los recursos didácticos y la reacción de los/as estudiantes, pero el foco hacia nosotros/as mismos/as se desdibujaba. Sin embargo, a través de las reflexiones realizadas en las parejas de trabajo y con el equipo en pleno, logramos comprender que el "sí mismo pedagógico" era el centro de esta experiencia.

[...] Voy de vuelta a casa con la sensación de que no comprendo bien este enfoque de trabajo. Honestamente el sentimiento es de decepción, tomo conciencia de que he estado resistiéndome a examinar mi propia experiencia 
y en realidad sigo atenta a la efectividad de mi trabajo en el aula. Tengo claro que debo dar un giro, pero no sé bien cómo hacerlo. Siento que es un desafío compartido por el equipo... (Notas de viaje, octubre 2016).

\section{Etapa 2. Apertura intersubjetiva}

Corresponde al momento en que asumimos el rol de "amigo/a crítico/a" y desarrollamos la relación de amistad crítica desde las preguntas desequilibrantes, provocadoras y reflexivas. Esto, indudablemente, fue un proceso marcado por la voluntad de construir espacios de confianza académica y de disponer del tiempo necesario para conversar con el otro de manera respetuosa y constructiva. En este punto, destacamos la experiencia formativa del diálogo con el otro, pues se transformó en nuestra "caja de resonancia" que nos interpelaba, haciendo eco en la propia práctica como formador de formadores.

[...] Acabo de cerrar la sesión de Skype y siento que esta forma de trabajar con mi amigo crítico, no solo ayuda a esclarecer aspectos de la práctica formativa, sino que muchas de las cuestiones que observo en el otro reflejan mi propia actuación y terminan interpelando mi propia práctica formativa. No se trata de un mismo contenido o práctica específica, sino que este análisis de la acción docente actúa como una caja de resonancia que se conecta con mis propias prácticas formativas y ahora empiezo a dudar sobre lo que hasta ahora tenía por seguro... que experiencia dura situación, pero transformadora a la vez... (Diario Docente, septiembre, 2016)

\section{Etapa 3. Toma de conciencia intersubjetiva}

Durante la investigación hubo instancias en que todos los participantes nos reunimos para tomar el pulso de lo vivido, auscultando en los significados emergentes de cada pareja. Volviéndose relevante la necesidad de observarnos de modo intersubjetivo/interinstitucional, es decir, detenernos a compartir desde la confianza, dialogar sobre cómo experimentábamos el proceso, cómo avanzábamos, incluso cómo nos entrampábamos en la mirada del 'sí mismo'. Tomar conciencia, desde la experiencia comunitaria, fue algo nuevo y revelador como equipo generador de conocimiento, y nos dio mayor seguridad, confianza y aliento para seguir avanzando.

[...] Siento que nuestro encuentro en Temuco ha sido un hito en esta experiencia de self-study, hay cuestiones que emergieron en la conversación que nos ayudaron a tomar consciencia de los aprendizajes que se han configurado en torno a esta experiencia de self-study. Nos damos cuenta que hemos aprendido en una perspectiva del self propiamente tal (Diario de viaje, abril, 2017). 


\section{Etapa 4. Aprendizaje Pedagógico}

La experiencia, inicialmente nos exigió hacernos conscientes de lo vivido, para luego extraer su potencial en términos de aprendizaje pedagógico. Comprender cómo la enseñanza puede enfrentarse de un modo diferente, sin duda, reformuló nuestro saber pedagógico sobre la enseñanza. Esta etapa logró ser del todo alentadora para el grupo, ya que, luego de un largo viaje de cara a nuestras propias prácticas, nos hacíamos consciente del propio aprendizaje que estábamos logrando.

[...] Por otra parte, hay aprendizajes que tienen relación con nuestras prácticas de enseñanza en la Universidad y que han sido posibles gracias a la metodología que nos insta a mantener una relación pensante y dialogante con nuestra propia actuación docente en el aula y fuera de ella. En el diálogo y en la escritura se anuncia y denuncia lo adquirido desde una experiencia mediada por las distintas miradas de los participantes. Se pone de manifiesto que esta experiencia tiene un alto sentido intersubjetivo, es decir, no se puede pretender llegar a aquello sin la interacción y cruce de todas las miradas de la experiencia compartida y eso, le otorga riqueza a esta opción interuniversitaria y colaborativa (Diario de viaje, Noviembre 2016).

\subsection{La construcción de la amistad crítica}

La figura del amigo crítico constituye un aspecto distintivo del self-study. Las formas de relación que construímos en este tipo de investigación con el amigo crítico son sostenidas en el diálogo, como espacio que va desarrollando la postura reflexiva y analítica en torno al quehacer del self (Pinnegar y Hamilton, 2009; Pinnegar y Quiles-Fernández, 2018). No obstante, el rol del amigo crítico constituyó un proceso que no estuvo exento de desafíos, aciertos y dificultades. Una distinción en este proceso fue el cambio paradigmático, ya que al inicio nos situamos desde un paradigma instrumental y por ello, esperábamos que nuestro amigo nos señalara qué hacer ante situaciones complejas o incidentes críticos, tal como queda consignado en el diario docente:

[...] Me encuentro en la oficina y honestamente, pensé que la amiga crítica podría haberme sugerido algunas ideas para enfrentar el incidente que se produjo en el aula, a propósito del rechazo a las narrativas semanales. Aunque le pregunté reiteradamente, no me sugirió nada, por el contrario me hizo más consciente de aspectos que no he contemplado sobre el empleo de este recurso de modo pedagógico. (Diario docente, enero 2017).

La mayoría esperaba que el amigo crítico hiciera un juicio de nuestra actuación y propusiera ideas para el mejoramiento, denotando un rol evaluativo y orientado a la mejora de la práctica, lo que ilustramos a continuación: 
[...] Honestamente, mi expectativa inicial era contar con un colega más experto, que me hiciera ver en qué fallaba y que además, propusiera sugerencia para mejorar. De a poco me fui dando cuenta que esto que espero obedece a una noción instrumental de este acompañamiento que hace que el otro tenga que hacer control y seguimiento de nuestro desempeño docente (Diario docente, septiembre, 2016).

Haciendo eco de nuestros estilos de amistad, intuíamos la necesidad de compartir tiempo, sostener conversaciones en torno a las experiencias formativas y de este modo, incrementar el conocimiento mutuo, ganando espacios de confianza para llegar al análisis crítico de la práctica pedagógica del otro. Esto no fue fácil de llevar a cabo y poco a poco consensuamos que el desafío era desarrollar cierta sensibilidad pedagógica para ver lo que habitualmente pasa desapercibido en términos formativos e interpelarnos con aquello.

[...] Semanalmente estoy tomando el tiempo para ver los videos de la clase y los comentarios que me envía por correo mi amigo del self-study. Yo como amiga crítica, me estoy dando cuenta que debo ir más allá de lo aparente. En lugar de ofrecer juicios valorativos, tengo que ayudarlo a develar lo que está a la base de lo que hace. Siento que él está valorando este ejercicio, parece que mis comentarios provocan cierto nivel de interpelación que mi amigo está valorando (Diario narrativo, abril 2017).

Un elemento clave de la amistad crítica fue reconocer que todos los formadores de formadores iniciábamos el análisis de la enseñanza a partir de algún suceso vivido en el aula. En otras palabras, escudriñamos "en lo vivido", develando nuevos significados con ayuda de los compañeros o compañeras. Incrementamos la comprensión de la experiencia formativa, constituyendo un punto de inflexión que nos llevó a re-pensar la enseñanza. Por consiguiente, se puede plantear que esta amistad crítica interpeló aspectos habituales de la enseñanza e instó a deliberar respecto de cambios necesarios para lograr una práctica formativa más pertinente.

[...] La amiga crítica comentó que en mi taller de práctica profesional, las preguntas llevaban a los estudiantes a autoevaluarse y en realidad ese tipo de cuestionamiento no estimula la reflexión más práctica a la que yo aspiraba. Eso me cuestionó profundamente y me instó a buscar la forma de cambiar este aspecto de mi práctica formativa (Diario, enero, 2017).

El espacio compartido "entre los amigos críticos", contempló encuentros presenciales y virtuales, comunicación oral y escrita. En el caso de académicos de una misma institución, dedicaron tiempo dentro de la jornada de trabajo para el ejercicio de esta amistad. Y los formadores de formadores de distintas instituciones mantuvieron una amistad crítica optando habitualmente por lo virtual. 
En esta experiencia, el rol del amigo crítico la asociamos a una representación que arranca habitualmente de una racionalidad técnica asociada a un experto que me dice qué debo hacer. Esto demanda inicialmente un juicio evaluativo desde el amigo crítico. Más temprano que tarde y de un modo dialéctico, nos fuimos dando cuenta que el rol del amigo crítico tiene una naturaleza de correspondencia. Una relación que lleva a desarrollar en el docente acompañado el crecimiento en la habilidad para interpelar, cuestionar y decidir, algo que en algún momento Ilamamos sensibilidad pedagógica. Este acompañamiento nos ayuda a decidir qué cambiar de la práctica de enseñanza para lograr cierto mejoramiento en la acción formativa.

\subsection{Aprendizajes como formadores de formadores}

Un aspecto relevante para nosotros fue reconocer la esencia del método investigativo del self-study. Sin duda, esto significó un gran esfuerzo, pues desde la lógica positivista enraizada en varios de nosotros, nos desplazamos a otro paradigma. El self-study se enmarca dentro de metodologías que adquieren una esencia subjetiva, por tanto más que una metodología se convierte en un enfoque distinto. Inicialmente nos planteamos propuestas de carácter técnico, que tenían un sentido de aplicación, recepción y reacción. La reflexión nos implicó salir del espacio jerárquico con nuestros estudiantes y con nuestros pares. Nos dimos cuenta de que la reflexión no era una actividad aislada y que un foco importante eran las interacciones que se generaban.

Si bien, en el equipo hubo diversas aproximaciones a los paradigmas de investigación, fue un desafío revisar el self, el "sí mismo"; dialogar el "sí-mismo pedagógico". Nos dimos cuenta que el self-study debe centrarse en esos cambios; aunque continuamos por un buen tiempo con cierto apego a los viejos paradigmas tan aceptados por la ciencia tradicional.

[...] Esta tarde me quedé pensando en lo dicho por mi amiga crítica, dijo que mis preguntas eran muy amplias y que no daba suficiente tiempo a los estudiantes para que pensaran sobre su propia experiencia. No sé qué me inquietó más, creo que definitivamente fue la segunda observación, es decir, la idea de que ellos reflexionen sobre su planificación. Entonces, mi actuación resultó ser una contradicción. Las preguntas eran amplias, era consciente de ello. En otras clases, la pregunta, habitualmente, confirma la comprensión conceptual, aquí debería desencadenar el cuestionamiento y en esa perspectiva optaba por preguntas amplias confiando que el propio estudiante focalizará en un asunto relevante para él o ella. Pero, veo que esto no resulta del todo cierto. (Diario profesional, 30 agosto, 2016). 
Conviene subrayar que, comprendimos que los caminos no siempre son Ilanos, pero que en esencia pueden ser menos intrincados si los transitamos con calma y vamos paso a paso avanzando, en la medida que fuéramos avanzando, madurando en el self. Tuvimos varias y variadas preguntas, pero en la medida en que avanzaron los encuentros con nuestros amigos críticos, comenzamos a convertirnos en creadores de nuestro propio proceso.

Las claridades y certezas que tuvimos en un comienzo a acerca del quehacer investigativo se difuminaron en un asunto que parecía a momentos cambiar de dirección. La tradición y la experiencia nos interpelan. Sin embargo, la reflexión constante y el intercambio dialógico con los amigos críticos, nos encauzaron con un mayor soporte. De esta forma disminuimos la sensación de estar perdidos en un paradigma inexplorado, al menos eso pensábamos.

El espacio generado en torno al self-study nos permitió como formadores de formadores vivenciar una reflexión crítica, la cual pasa a ser pública, y se convierte en colectiva en la medida que somos capaces de cotejar e interpretar junto a otros nuestras problemáticas, en una perorata que nutre la formación y que, en definitiva, nos acerca a profesionalizar nuestra disciplina, la enseñanza.

\subsection{Aprendizajes de los/as estudiantes}

Consideramos que el foco más importante de la transformación de nuestras prácticas, consiste en el avance colectivo hacia volvernos conscientes y a comprometernos con los resultados de aprendizaje que muestran nuestros estudiantes. El self-study también nos permitía mirar nuestra propia práctica en función del aprendizaje del estudiante, por cuanto comenzamos a relevar su voz, a interpretarla y reconocer en ellos nuestra transformación.

Como una forma de darnos cuenta acerca de cómo nuestras propias reflexiones las íbamos transfiriendo, registramos algunos aprendizajes por parte de nuestros estudiantes. La reflexión en torno a nuestro propio "sí mismo" iba poco a poco generando también un cambio en la relación de nuestros estudiantes con sus propios procesos reflexivos. Reflexión lleva a reflexión

[...] A veces las preguntas de la profe no sé a qué se refieren y yo contesto y tengo la impresión que no doy con lo que ella me está preguntando y eso, a veces me pone un poco nerviosa y no me gusta. Miro a mis compañeros y ella, formula otra pregunta... podría esperar un poco... no hay mucho tiempo para pensar y eso... (Narrativa Paula, septiembre, 2016)

Profesora: Oye ¿Qué aspecto de la clase nos preocupa? En la perspectiva del análisis que estamos desarrollando, ¿Qué nos inquieta? 
Estudiante: Yo creo que no sabemos si los estudiantes están aprendiendo. O sea como que se centra mucho en la trasmisión del conocimiento. "Yo digo y los estudiantes anotan y entonces, yo les planteo preguntas y ellos responden lo que yo quiero escuchar.

Profesora: Ya, y entonces, ¿Qué significado subyace a esa práctica docente?

Estudiante: Evidentemente se trata de un modelo de enseñanza tradicional, en que se transmiten verdades en las clases de ciencias. Bueno, a mí eso, me da rabia porque justamente este tipo de clase es la que más critiqué $y$ ahora veo que yo hago lo mismo.

Reservamos el espacio declarado por los estudiantes para acoger preguntas e inquietudes que llevan a repensar y analizar lo acontecido con una mirada pedagógica y a construir desde ahí un saber docente. Definitivamente, el dilema no estaba en cómo trabajar la reflexión con un determinado dispositivo; sino más bien, tratábamos de construir una nueva relación reflexiva con los/as estudiantes.

\section{Discusión}

Ante todo subrayar que esta etapa fue, con aún más cuidado, una construcción colectiva de todos los integrantes de este estudio. La discusión final de los hallazgos nos brindó la posibilidad de concluir en torno a cuatro cuestiones: al empleo del método, la construcción de relaciones a través de la amistad crítica, los aprendizajes como formadores de formadores y los resultados desde los estudiantes

Respecto del método self-study vivimos un proceso que no estuvo exento de tensiones en torno a reconocer que el objeto de estudio era el "sí mismo pedagógico" (Contreras, 2016; Loughran y Russell, 2016; Vannassche y Kelchtermans, 2015, 2016). Además, la dificultad de asumir que este método se configura en torno a relaciones intersubjetivas que constituyen el sustrato para evaluar nuestro trabajo formativo, su interpelación y transformación. Esto nos Ileva a promover esta metodología para favorecer la autoformación basada en la relación intersubjetiva (Lunenberg, Zwart y Korthagen, 2010) como una manera de modelar la enseñanza basada en el análisis de la propia práctica (Lunenberg y Samaras (2011). De igual modo, fuimos capaces de identificar etapas en el proceso de self-study; resistencia al foco en el "sí mismo", apertura a lo intersubjetivo (amistad crítica), la toma de conciencia intersubjetiva (proyecto interinstitucional) y finalmente, lo que denominamos el aprendizaje pedagógico. Respecto a la forma en que nos constituimos como grupo de self-study, esto quedó ampliamente escrito en un artículo anterior (Silva-Peña et al., 2017). 
En un self-study reciente, Pinnegar y Quiles-Fernández (2018) narran cómo la investigación se transforma en un proceso de construcción de relaciones entre los participantes. En nuestro caso, vemos que el self-study desarrollado fue una buena vía de construcción de relaciones entre nosotros, como colegas, académicos, formadores de formadores e investigadores de nuestra práctica. Para esto, fue crucial la configuración del rol de amigos críticos (Schuck y Russell, 2016), destacando su rol de "caja de resonancia" a través de preguntas desafiantes, provocativas o interpeladoras que, apoyaban la reconstrucción de la experiencia y el aprendizaje profesional.

Es importante transparentar que observamos una tensión al configurarse el rol del amigo crítico, pues inicialmente la expectativa era de conducción y en este sentido, cobra valor la tensión que evidencia Berry (2016) entre valorar la experiencia y reconstruirla para levantar conocimiento práctico (Van Manen, 2003). De este modo, el grupo evolucionó en las funciones del amigo crítico desde un rol conducción/evaluador a un rol de sensibilización/ interpelación y deliberación. Además, identificamos condiciones de carácter personal así como características del contexto institucional que favorecieron la amistad crítica, coincidiendo con Schuck y Russell (2016) en cuanto a que esta amistad beneficia a la díada: docente y amigo crítico. Pero el proceso de convertirse en un amigo que, interpele o ayude a deliberar, es de construcción lenta, consolidándose en la medida que el diálogo se hace más continuo en frecuencia, honestidad y reflexión.

En cuanto a los aprendizajes como formadores de formadores queremos subrayar que ahora somos más conscientes que el self-study desencadena construcción de saberes docentes desde la práctica de la enseñanza (Tardif, 2004) y estos conocimientos se definen como aquellos que irrumpen mediados por un análisis reflexivo sobre la propia experiencia, y por eso, nos atrevemos a plantear que el self-study promueve reflexión en y sobre la acción formativa en contexto de formación de docente (Schön, 1992).

Quienes participamos del proceso de self-study teníamos bagaje en el uso de los Ilamados "dispositivos" o recursos para estimular una experiencia de reflexión en la formación inicial docente (Nocetti, 2016; Nocetti y Medina, 2017; Salgado-Labra y Silva-Peña, 2009; Silva-Peña, Valenzuela y Santibáñez, 2008). Sin embargo, el desarrollo del self-study nos permitió comprender con mayor profundidad nuestra acción formativa y desencadenó la necesidad de transformar nuestra actuación; evidenciando la coherencia con lo que distingue al self-study en términos de ser un examen situado de la propia práctica que lleva a una mayor comprensión de la acción y la búsqueda de la transformación de esta (Cornejo, 2016, Loughran y Russell, 2016, Russell, Fuentealba e Hirmas, 2016). 
Pensamos que el self-study es una construcción transformadora, y que a pesar de iniciar junto a los amigos críticos desde el predominio de una lógica instrumental, logramos movilizarnos a un estadio de reflexión que promovió no solo nuestras racionalidades, sino también la de los estudiantes con quienes compartimos.

Dado que el self-study se orienta a la mejora de la enseñanza, esta investigación nos instó a construir un saber práctico que emerge del diálogo pensante con las situaciones vividas en el Taller de Práctica (Contreras, 2013, Tardif, 2004), estimulando no solo una práctica reflexiva en los formadores de formadores, sino también impulsando a explicitar los saberes pedagógicos que se van construyendo en el proceso de esta investigación con carácter formativo (Berry, 2016) y que la mayoría de las veces se mantienen excluidos de los espacios de discusión académica. Por otra parte, a pesar de nuestras aspiraciones, la racionalidad técnica predomina en nuestras prácticas docentes y limita las oportunidades para ofrecer una experiencia temprana de análisis reflexivo deliberativo/crítico (Nocetti, 2016).

Una reflexión compartida sobre el self-studyfue reconocer decisiones pedagógicas que se amparan en prácticas previas aparentemente efectivas. Hay mucho de intuitivo y una falta de consciencia de que esto es así. Los docentes no tenemos suficientes instancias para examinar nuestras propias prácticas en colaboración con otros pares formadores que nos exhorten a cuestionar lo que se ha automatizado o dado por cierto y efectivo. Indudablemente, evidenciar aquello, es el primer paso para una práctica transformadora y en ese sentido, el self-study tiene un alto potencial formativo.

Reconocemos algunas limitaciones en el trabajo realizado. Entre estas se encuentran ciertos factores personales y condiciones institucionales que impidieron un trabajo más sistemático, especialmente, a nivel del equipo interuniversitario. Además, consideramos necesario incluir al estudiantado de modo más activo en la retroalimentación sobre nuestras transformaciones en la práctica.

A modo de proyección, convendría desarrollar un estudio con mayor alcance para establecer si las fases vividas en el proceso llevado a cabo son compartidas por otros formadores y, a partir de ello, levantar sugerencias para la implementación de un nuevo proyecto self-study. Así mismo, explorar sobre las habilidades interpersonales y las condiciones institucionales que favorecen una amistad crítica, sondeando las diferencias entre la amistad organizada en diadas o en comunidades de amigos críticos. 


\section{Agradecimientos}

Este trabajo ha sido financiado por la OEI (Organización de Estados Iberoamericanos) a través del proyecto: "Self-study colaborativo sobre prácticas de formación docente".

\section{Referencias bibliográficas}

Alliaud, A. y Suárez, D. (2011). El saber de la experiencia. Narrativa, investigación y formación docente. Buenos Aires: CLACSO.

Altet, M. (2010). La competencia del maestro profesional o la importancia de saber analizar las prácticas. En: Paquay, L. et al. (Coords.). La formación profesional del maestro. Estrategias y competencias (pp. 33-54). México: Fondo de Cultura Económica.

Alvarez-Gayou, J. (2013). Como hacer una investigación cualitativa. Fundamentos y metodología. México: Paidós.

Berry, A. (2016). Tensiones en la reconceptualización del conocimiento del formador de formadores: explorando la tensión en revalorar y reconstruir la experiencia. En: Russell, C. Hirmas y R. Fuentealba (Eds.), Formadores de formadores, descubriendo la propia voz a través del Self-Study (pp. 77-95). Santiago: OEI. Recuperado de https://bit.ly/2UmR8c9

Bisquerra, R. (2014). Metodología de la investigación educativa, $4^{\circ}$ edición. Madrid: La Muralla.

Chacón, M. y González, A. (2008). Las estrategias de enseñanza reflexiva en la formación inicial docente. Educere, 12(41), 277-287.

Clandinin, D. J. y Connelly, F. M. (2000). Narrative inquiry: Experience and story in qualitative research. San Francisco: Jossey-Bass.

Connelly F. M. y Clandinin, D. J. (1990). Stories of Experience and Narrative Inquiry. Educational Researcher, 19(5), 2-14.

Contreras D. J. y Pérez de Lara, N. (2010). Investigar la experiencia educativa. Madrid: Morata.

Contreras, J. (2011). El lugar de la experiencia. Cuadernos de pedagogía, 417, 60-63.

Contreras, J. (2013). El saber de la experiencia en la formación inicial del profesorado. Revista Interuniversitaria de Formación del Profesorado, 78 (27, 3), 125-136. Recuperado de http://bit.ly/2QRYLoF

Contreras, J. (2016). Relatos de experiencia, en busca de un saber pedagógico. Revista Brasileira de Pesquisa (Auto) Biográfica, 01 (01), 14-30. Recuperado de http://bit.ly/37DLzK6

Cornejo, J. (2016). El Self-Study de la práctica de los formadores de futuros profesores: bases teórica, características y modalidades metodológicas. En: Russell, T., Fuentealba. e Hirmas, C. (2016). Formadores de formadores, descubriendo la propia voz a través del Self-Study (pp. 25-63). Chile: BuK\&Editora. Recuperado de https://bit.ly/2UmR8c9

Correa, E., Chaubet, P., Collin, S. y Gervais, G. (2014). Desafíos metodológicos para el estudio de la reflexión en contexto de formación docente. Estudios pedagógicos (Valdivia), 40(Especial), 71-86. Recuperado de http://bit.ly/33pKFNL 
Crotty, M. (1998). The foundations of social research: Meaning and perspective in the research process. London: Thousand Oaks; New Dehli: Sage.

Del Barrio, A. (2014). De la reflexión personal a la reflexión crítica en equipo: aprendizaje permanente en equipo, un modelo para una práctica reflexiva y democrática en la primera infancia. Revista Española de Educación Comparada, 23, 163-181.

Guba, E. G. y Lincoln, Y. S. (1994). Competing paradigms in qualitative research. Handbook of Qualitative. London: Sage.

Guerra, P. (2009). Revisión de experiencia de reflexión en la formación inicial de docentes. Estudios Pedagógicos, 35(2), 243-260. http://dx.doi.org/10.4067/S0718$\underline{07052009000200014}$

Grundy, S. (1987). Producto praxis del curriculum (2a ed.). Madrid: Morata.

Hamilton, M. L. (1998). Reconceptualizing Teaching Practice: Self-Study in Teacher Education. London: Falmer Press.

Hirmas, C. y Cortés, I. (2015). Investigaciones sobre formación práctica en Chile: tensiones y desafíos. Santiago: OEI.

Korthagen, F. (2010). La práctica, la teoría y la persona en la formación del Profesorado. Revista Interuniversitaria del Profesorado, 68, 83-101. Recuperado de http://bit.Iy/2skdNt9

Loughran, J. (2007). Researching teacher education practices: Responding to the challenges, demands, and expectations of Self-Study. Journal of Teacher Education, 58(1), 12-20. https://doi.org/10.1177/0022487106296217

Loughran, J. y Russell, T. (2016). Comenzando a entender la enseñanza como disciplina. En: T. Russell, R. Fuentealba, e C. Hirmas (2016). Formadores de formadores, descubriendo la propia voz a través de/ Self-Study (65-115). Santiago: OEI. https://bit.ly/2UmR8c9

Luneberg, M., Samaras, A. (2011). Developing a pedagogy for teaching Self-Study research: Lessons learned across the Atlantic. Teaching and Teacher Education. 27, 841-850. https://doi.org/10.1016/j.tate.2011.01.008

Lunenberg, M. Zwart, R. y Korthagen, F. (2010). Critical issues in supporting Self-Study. Teaching and teacher Education. 26 (6), 1280-1289.

Nocetti, A. (2016). Experiencia de Reflexión de estudiantes de pedagogía en Educación media en Biología y Ciencias Naturales en las asignaturas de práctica pedagógica y profesional en una universidad de la región del Biobío, Chile (Tesis para optar al grado de Doctor en Educación y Sociedad). Universidad de Barcelona, España.

Nocetti, A. y Medina, J. (2017). Ciclo Reflexivo de Aprendizaje Docente (CRAD) y la comprensión de aspectos didácticos de la acción docente. En: J. Osorio y M. Gloël (Coords.). La didáctica Como fundamento de la práctica profesional docente: tendencias, enfoques y avances. Concepción, Chile: Universidad Católica de la Santísima Concepción.

Nocetti, A. y Medina, J. (2018). Condiciones que desencadenan la reflexión docente en el futuro profesor durante sus prácticas de formación. Revista Espacios, 39(15).

Nocetti, A. y Medina, J. (2019). Significados de reflexión sobre la acción docente en el estudiantado y sus formadores en una universidad chilena. Revista Educación, 43(1). Recuperado de http://bit.ly/2qM1aqi

OCDE (2004). Chile. Revisión de las políticas Nacionales de Educación. París: OCDE. 
OCDE/Banco Mundial (2009). Revisión de Políticas Nacionales de Educación: la Educación Superior en Chile. París: OCDE.

Paquay, L., Altet, M., Charlier, E. y Perrenoud, P. (2010). La formación profesional del maestro. México: Fondo de Cultura Económica.

Perrenoud, P. (2004). Desarrollar la práctica reflexiva en el oficio de enseñar: Profesionalización y razón pedagógica. Madrid: Grao.

Perrenoud, P. (2010). El trabajo sobre el habitus en la formación de maestros. Análisis de las prácticas y toma de conciencia. En: Paquay, L., Altet, M., Charlier, E., Perrenoud, P. (2010). La formación profesional del maestro. Estrategias y Competencias (pp. 265-304). México: Fondo de Cultura Económica.

Pinnegar, E. y Quiles-Fernández, E. (2018). A Self-Study of Researcher Relationships with Research Participants. Studying Teacher Education, 14(3), 284-295. https://doi. org/10.1080/17425964.2018.1541287

Pinnegar, S. y Hamilton, M. L. (2009). Self-Study of practice as a genre of qualitative research: Theory, methodology, and practice. Netherlands: Springer.

Pozo, J. (2012). Competencias profesionales. Herramientas de evaluación: el portafolios, la rúbrica y las pruebas situacionales. Madrid: Narcea.

Rodríguez, G., Gil, J. y García, E. (1999). Metodología de la Investigación Cualitativa. España: Aljibe.

Russel, T. (2012). Cambios paradigmáticos en la formación de profesores: Peligros, trampas y la promesa no cumplida del profesional reflexivo. Rencontres on Education, 13, 71-91

Russell, T., Fuentealba, R., e Hirmas, C. (2016). Formadores de formadores, descubriendo la propia voz a través del Self-Study. Chile: OEl. https://bit.ly/2UmR8c9

Salgado, I. y Silva-Peña, I. (2009). Desarrollo profesional docente en el contexto de una experiencia de Investigación-acción. Paradigma, 30(2), 63-74.

Samaras, A. (2010). Self-study teacher research: Improving your practice through collaborative inquiry. London: Sage.

Sandín, M. (2003). Investigación cualitativa en educación. Fundamentos y tradiciones. Madrid: Mac-GrawHill.

Sanjurjo, L. (2005). La Formación práctica de los docentes. Reflexión y acción en el aula. Buenos Aires: Homo Sapiens.

Schön, D. (1992). La formación de profesionales reflexivos. Hacia un nuevo diseño de la enseñanza y el aprendizaje en las profesiones. Barcelona: Paidós.

Schuck, S. y Russell, T. (2016). Self-Study, amistad crítica y las complejidades en la formación de profesores. En: T. Russell, R. Fuentealba y C. Hirmas (2016). Formadores de formadores, descubriendo la propia voz a través del Self-Study (pp. 117-147). Chile: OEI. https://bit.Iy/2UmR8c9

Silva-Peña, I. (2007). Condiciones y contextos en torno al Desarrollo Profesional Docente producido a través de la Investigación-Acción. (Tesis para optar al grado de Doctor en Ciencias de la Educación). Pontificia Universidad Católica de Chile, Chile. 
Silva-Peña, I., Driedger-Enns, L., Huber, J. y Houle, S. (2016). Relaciones dentro del aula: Hacia la construcción de comunidad en Educación Parvularia. Presentado en XXIII Encuentro Nacional de Investigadores en Educación, 9 al 11 de Noviembre. Valparaíso, Chile.

Silva Peña, P. Moreno, D. Santibáñez, M. Gutiérrez, M. Flores, C. Orrego y A. Nocetti. (2017). Self-Study como proceso de formación de formadores/as: reflexiones en medio del camino. Ed UCSH.

Silva-Peña, I., Valenzuela, J. y Santibañez, M. (2008). Representaciones sociales sobre la reflexión docente en estudiantes del último año de formación inicial en Educación General Básica. En: Cornejo, J y Fuentealba, R. (2008). Prácticas reflexivas para la formación profesional docente: ¿qué las hace eficaces?( pp.29-53). Chile: Ediciones Universidad Católica de Chile.

Silva-Peña, I. y Paz-Maldonado, E. (2019). Una reflexión acerca de la indagación narrativa autobiográfica en formadores/as de docentes para la justicia social. Perspectiva Educacional, 58(2), 169-189. http://dx.doi.org/10.4151/07189729-vol.58-iss.2art.953

Tardif, M. (2004) Los saberes del docente y su desarrollo profesional. Vol. 97. Narcea Ediciones.

Vanassche, E. y Kelchtermans, G. (2016). Facilitating Self-Study of teacher education practices: toward a pedagogy of teacher educator professional development. Professional Development in Education, 42(1), 100-122. https://doi.org/10.1080/1941525 $\underline{7.2014 .986813}$

Vanassche, E. y Kelchtermans, G. (2015). The state of the art in Self-Study of Teacher Education Practices: a systematic literature review. Journal of Curriculum Studies, 47(4), 508-528. https://doi.org/10.1080/00220272.2014.995712

Van Manen, M. (2003). Investigación educativa y experiencia vivida. Barcelona: Idea Book.

Zabalza, A. (2005). Diarios de clase. Un instrumento de investigación y desarrollo profesional. Madrid: Narcea. 